\title{
Robust detection of ionospheric scintillations using MF-DFA technique
}

\author{
Sridhar Miriyala ${ }^{1 *}$, Padma Raju Koppireddi ${ }^{2}$ and Srinivasa Rao Chanamallu ${ }^{3}$
}

\begin{abstract}
The performance of Global Navigation Satellite System (GNSS) receivers is limited by the ionospheric scintillation effects that cause signal degradation due to refraction, reflection and scattering of the signals. Hence, there is a need to develop an ionospheric scintillation detection technique for robust GNSS receivers. In this paper, a new algorithm based on multifractal detrended fluctuation analysis (MF-DFA) is proposed for detecting the ionospheric irregularities. The ionospheric and scintillation GNSS data recorded at Koneru Lakshmaiah (KL) University, Guntur, India, was considered for the analysis. The carrier to noise ratio $\left(C / N_{0}\right)$ time series data of GNSS satellite vehicles that are affected due to scintillations was decomposed using adaptive time-frequency methods like empirical mode decomposition (EMD), ensemble empirical mode decomposition (EEMD) and complementary ensemble empirical mode decomposition (CEEMD). It was observed that the CEEMD method combined with MF-DFA provides better results as compared to the EMD and EEMD techniques.
\end{abstract}

Keywords: Global positioning system; Radio wave propagation; Complementary ensemble empirical mode decomposition; Multifractal detrended fluctuation analysis; Hurst exponent

\section{Findings \\ Introduction}

Ionospheric scintillation is one of the predominant propagation impairments at L-band frequencies that persist because of the existence of irregularities in the ionosphere due to refraction, reflection and scattering of the radio signals (e.g. De Paula et al. 2003; Iyer et al. 2006; Cherniak et al. 2014). When a radio wave passes through the ionosphere, the irregularities distort the wavefront and as the wave moves towards the ground, phase mixing occurs creating a diffraction pattern on the ground. This diffraction phenomenon was determined by the size and strength of the ionospheric irregularities (Yeh and Liu 1982). The diffraction mechanism gives rise to amplitude scintillations which occur mainly due to smallscale irregularities when phase variations are small. However, due to large scale irregularities, the phase fluctuations are dominant and the wave becomes noncoherent thereby focusing and defocusing of the rays is not possible (Wernik et al. 2004). As the interference

\footnotetext{
* Correspondence: sridhar.m@kluniversity.in

'Department of ECE, KL University, Guntur, India

Full list of author information is available at the end of the article
}

mechanism is not valid under such conditions, amplitude scintillations do not increase further and phase scintillations become significant (Wernik et al. 2004). Amplitude and phase scintillations degrade the Global Navigation Satellite System (GNSS) receiver's tracking performance (e.g. Kintner et al. 2007; Aquino and Sreeja 2013). In order to improve the positional accuracy and availability of GNSS receivers, several methods have been proposed to detect the ionospheric scintillations (e.g. Suman et al. 2004; Pullen et al. 2009). A real-time ionospheric scintillation model, which determines the automatic threshold for different scintillation signals using the Neyman-Pearson detector, has been implemented (Venkata Ratnam et al. 2015). Mushini et al. (2012) proposed a wavelet-based detrending technique for detrending GPS signals. Wavelet detrending technique reveals local features of the signals as compared to Butterworth detrended filter (Mushini et al. 2012). Empirical mode decomposition (EMD) and ensemble empirical mode decomposition (EEMD) methods have been found to be suitable for analysing nonstationary signals (Wang et al. 2012). Multifractal detrended fluctuation analysis (MF-DFA) has been 
successfully implemented in medical and geophysics applications to investigate the self similarities and long-range correlations in the signals (e.g. Kantelhardt et al. 2002; Tanna and Pathak 2014).

The multifractality nature of the ionospheric time series data due to long-range correlations can be analysed using the MF-DFA technique. With this technique, the non-linear properties and complexity of random ionospheric irregularities are determined. In this letter, a novel algorithm, complementary ensemble empirical mode decomposition (CEEMD)-MF-DFA, was proposed and implemented to extract the noise components of the GPS signal. The results obtained using the CEEMD-MFDFA technique was compared and validated with the wavelet, EMD-MF-DFA and EEMD-MF-DFA techniques.

\section{CEEMD-MF-DFA algorithm}

EMD is an adaptive technique used to analyse nonlinear and non-stationary signals. The major advantage of this time-frequency data analysis method lies in deriving the basis functions from the characteristics of the signal, whereas the basis functions in wavelets are predefined based on the mother wavelet used (Mallat 1999). Sifting process is used to generate the intrinsic mode functions (IMF), and a residue which, when added, will give the original signal reconstruction. The residue represents the trend of the signal and cannot be decomposed further. To achieve the effective functioning of EMD, the differences between the frequencies and amplitude must be sufficient for decomposition analysis, which otherwise leads to the limitation known as mode mixing (Huang et al. 1998).

Hence, to avoid the consequences of a mode-mixing problem, an improved EMD algorithm has been proposed known as EEMD. In this method, a Gaussian white noise is added before decomposition to minimise the effects of mode mixing in the EMD process. However, the inclusion of white noise with inappropriate amplitudes will generate a different number of modes that contains the components not related to the signal. Also, it introduces the residual of noise in the reconstructed signal (Wu and Huang 2009).

To overcome the problems of the EEMD method, CEEMD has been proposed in which positive and negative white noises are added to the data. Hence, two sets of ensemble IMFs are produced, and the reconstructed signal can be obtained by finding the mean of these IMFs. In CEEMD, residual of the added white noise contained in the IMFs is eliminated completely (Yeh et al. 2010). IMFs of EMD, EEMD and CEEMD have been derived for preparing inputs of the MF-DFA techniques.

The algorithm of MF-DFA was initiated with the calculation of average for each IMF $I(i)$ of $C / N_{0}$ time series data, and the integrated signal $y(i)$ was obtained by removing the average from the IMF; it is calculated by the following equation:

$$
y(i)=\sum_{l=1}^{i}\left[I(l)-\frac{1}{M} \sum_{k=1}^{M} I(k)\right]
$$

where $M$ is the length of the time series.

Next, the entire integrated time series of IMF was separated into $M_{s}$ segments which are non-overlapping where $M_{s}=M / s$ and $s$ is the length of the segment. For the present case, the entire time series was divided into 20 segments, where $s=1000$ is the length of the segment. In general, the length of the segment must be chosen to avoid computation error in fluctuation function $F(q)$. The complete procedure of this step was repeated from the opposite end as $M$ is not a multiple of length $s$, and hence, $2 M_{s}$ segments were obtained. In the next step, the least square fit was performed in each segment and the variance was calculated for the segments $u=1, \ldots, M_{s}$ using the equation below:

$$
F^{2}(s, u)=\frac{1}{s} \sum_{i=1}^{s}\left\{y[(u-1) s+i]-y_{u}(i)\right\}^{2}
$$

For the segments $u=M_{s}+1, \ldots, 2 M_{s}$, the variance was obtained as

$$
F^{2}(s, u)=\frac{1}{s} \sum_{i=1}^{s}\left\{y\left[M-\left(u-M_{s}\right) s+i\right]-y_{u}(i)\right\}^{2}
$$

To evaluate the effect of fluctuations of various amplitudes of IMF signals and on various time scales, the $q$ th order fluctuation for $q \neq 0$ is given by (Kantelhardt et al. 2002)

$$
F_{q}(s)=\left\{\frac{1}{2 M_{s}} \sum_{u=1}^{M_{s}}\left[F^{2}(s, u)\right]^{\frac{q}{2}}\right\}^{\frac{1}{q}}
$$

$q$ orders between -5 and 5 are more suitable for avoiding errors in multifractal spectrum (Ihlen 2012). The scaling behaviour of the fluctuation function can be revealed from the variation of $F_{q}(s)$ with the segment size $s$ for different orders on a $\log -\log$ scale that obeys the power law given by

$$
F_{q}(s) \sim s^{h(q)}
$$

where $h(q)$ is the scaling exponent known as generalised Hurst exponent (Hurst 1951). For a multifractal time series data, there exist a number of Hurst exponents for different $q$ orders of fluctuation. For positive values of $q$, Hurst exponent characterises the scaling nature of the segments with large variations, and for negative $q$ values, $h(q)$ indicates the scaling performance of the segments 
with small variations exhibiting the multiscaling features of the signal considered (Kantelhardt et al. 2002).

The Hurst exponent value is a key parameter in estimating the threshold, and the IMFs with $h(q)$ greater than the threshold are considered for reconstruction of the signal; the remaining IMFs constitute the scintillation components. As the scintillation noise was assumed as white noise, Hurst exponent of 0.5 was considered for analysis. In the case of EMD-MF-DFA, to reduce modemixing effects, a threshold $\varphi=H+0.2=0.7$ was used for identifying the noisy IMFs. For the EEMD-MF-DFA and CEEMD-MF-DFA techniques, a threshold $\varphi=H=0.5$ was considered as a mode-mixing problem does not exist in these methods (Mert and Akan 2014).

\section{Results and discussion}

Koneru Lakshmaiah (KL) University, Guntur $\left(16.31^{\circ} \mathrm{N}\right.$, $80.37^{\circ} \mathrm{E}$ ), falls under the transition zone of equatorial ionisation anomaly crest and trough of low latitude regions. Pseudo random noise (PRN) code 15 satellite signal (29 June 2013) was applied with the CEEMD-MFDFA technique. To avoid multipath effects, the signal with elevation angles greater than $30^{\circ}$ was considered. The maximum amplitude scintillation index $\left(S_{4}\right.$ index) value observed was 0.85 at $23.28 \mathrm{~h}$ (IST). The corresponding decrease in carrier to noise ratio $\left(C / N_{0}\right)$ to $31 \mathrm{~dB}-\mathrm{Hz}$ indicates the presence of non-linear irregularities in the ionosphere.

Figure 1 shows the 12 IMFs generated using the CEEMD technique for $C / N_{0}$ values of PRN 15 on 29 June 2013 sampled at $1 \mathrm{~Hz}$ rate. A white noise of 0.1 standard deviation was added to the GPS signal in the EEMD and CEEMD techniques before decomposing for solving the mode-mixing problem. Due to the inclusion of complementary noise components, the modes generated using CEEMD matched with the inherent characteristics of the signal. This feature has been considered in the CEEMD-MF-DFA technique. It is evident that the CEEMD method decomposed the signal into finer components than the EMD and EEMD methods for the given ensemble number of iterations. The IMFs obtained contained both significant signal components as well as scintillation components, whereas the residue indicated the trend of the $C / N_{0}$ signal. To detect the scintillations, the MF-DFA technique was applied to each and every IMF to calculate the values of Hurst exponent $(H)$.

The MF-DFA algorithm separated the IMFs of each decomposition method into non-overlapping segments from which multifractal detrended fluctuation $F(q)$ was calculated using (4). The input parameters to the MFDFA algorithm were segment size, fluctuation order $q$ and trend order $m$. These parameters were selected to obtain the optimised performance of the algorithm (Kantelhardt et al. 2002). For a given IMF, the Hurst

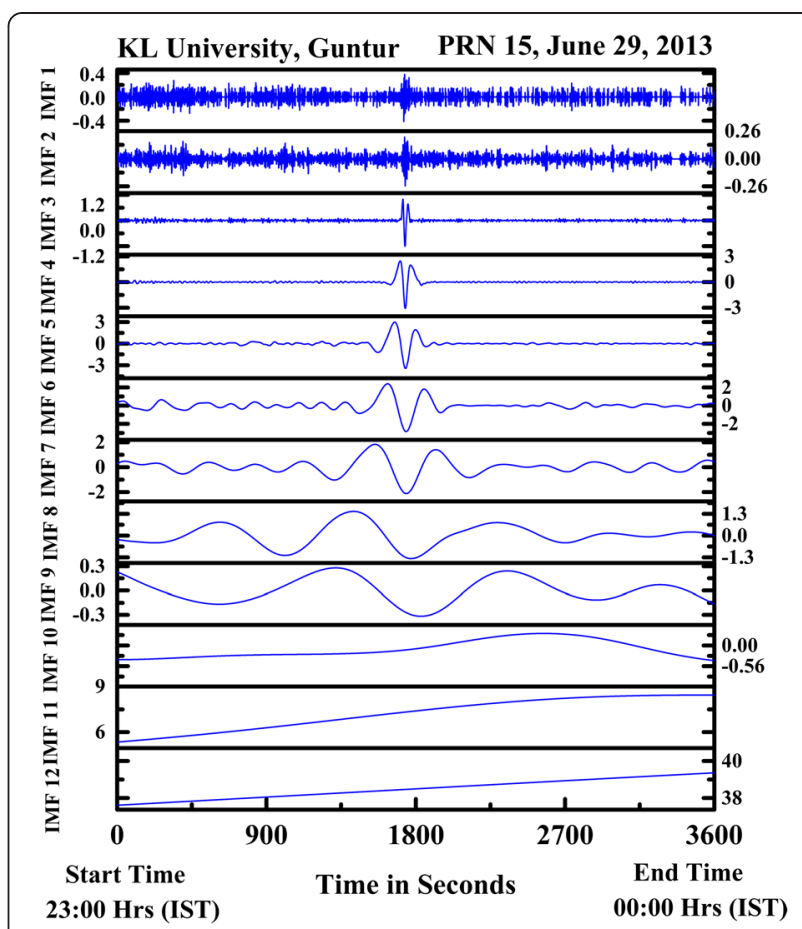

Fig. 1 The 12 IMFs generated using the CEEMD technique for C/No values of PRN 15 on 29 June 2013 sampled at $1 \mathrm{~Hz}$ rate

exponent will be different for different $q$ orders; hence, the amount of scintillation noise that can be mitigated is significantly different as the Hurst exponent may fall either below or above the threshold. Hurst exponents for the orders $q=1, q=3$ and $q=5$ were calculated for the IMFs of the CEEMD technique. It was identified that the first four IMFs were noises as their Hurst exponents fell below the threshold for orders $q=1$ and $q=3$. On the other hand, the first five IMFs constituted the scintillations when $q=5$, indicating a better reduction of noise. Hence, in this analysis, a linear trend was followed with $m=1$ and order $q=5$ was considered to represent intense amplitude scintillations over a short time period. Figure 2 indicates the variation of generalised Hurst exponent for the IMFs obtained using the EMD, EEMD and CEEMD methods for order $q=5$. In the case of EMD-MF-DFA, as the threshold was fixed at 0.7, the last $8 \mathrm{IMFs}$ were used for the reconstruction of $C / N_{0}$ signal, whereas IMFs 6 to 12 were considered for the EEMD/CEEMD-MF-DFA methods, resulting in an improved performance.

By summing up the IMFs with $H$ values below the threshold, we can obtain the amount of scintillation noise from the data. Figure 3 shows the results of the CEEMD-MF-DFA method in reducing the noise from the GPS signal. The noise of about $8.20 \mathrm{~dB}-\mathrm{Hz}$ was extracted from the signal using the proposed method for PRN 15 on 29 June 2013 at 23.28 h (IST) when 


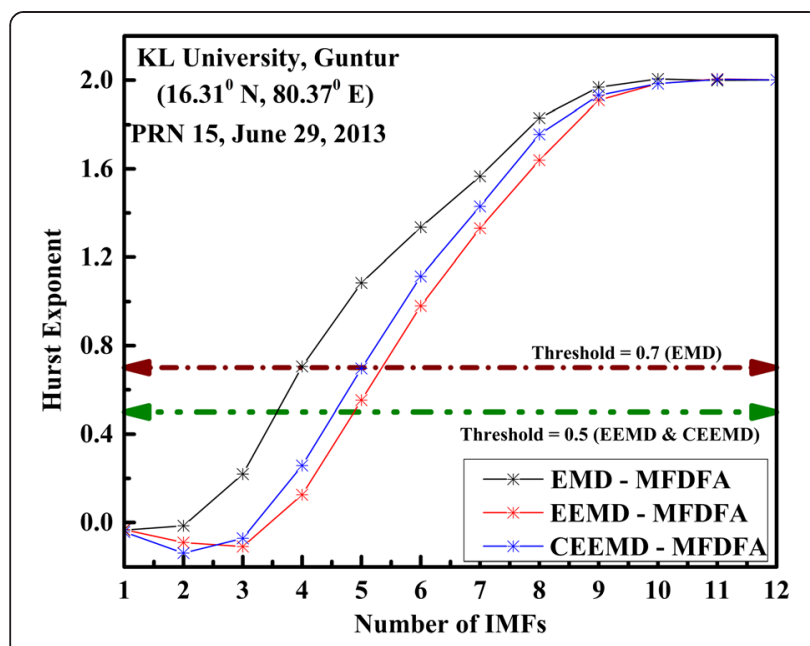

Fig. 2 The variation of generalised Hurst exponent for the IMFs obtained using the EMD, EEMD and CEEMD methods for order $q=5$

compared to $7.01 \mathrm{~dB}-\mathrm{Hz}$ with the EEMD-MF-DFA method. The amount of noise reduced using wavelets and the EMD-MF-DFA methods was 0.44 and $7.64 \mathrm{~dB}-\mathrm{Hz}$, respectively. It is evident from the results that detection of scintillation noise is better with the CEEMD-MFDFA method than the existing techniques due to inclusion of complementary noise that reduces the residual of the noise. Since threshold of 0.7 was used for the EMD method to compensate the mode-mixing problem, the detection of noise was better with the EMD than with the EEMD technique. During the post sunset period on 29 June 2013, where $C / N_{0}$ values of PRN 15 had been reduced significantly because of amplitude scintillations,

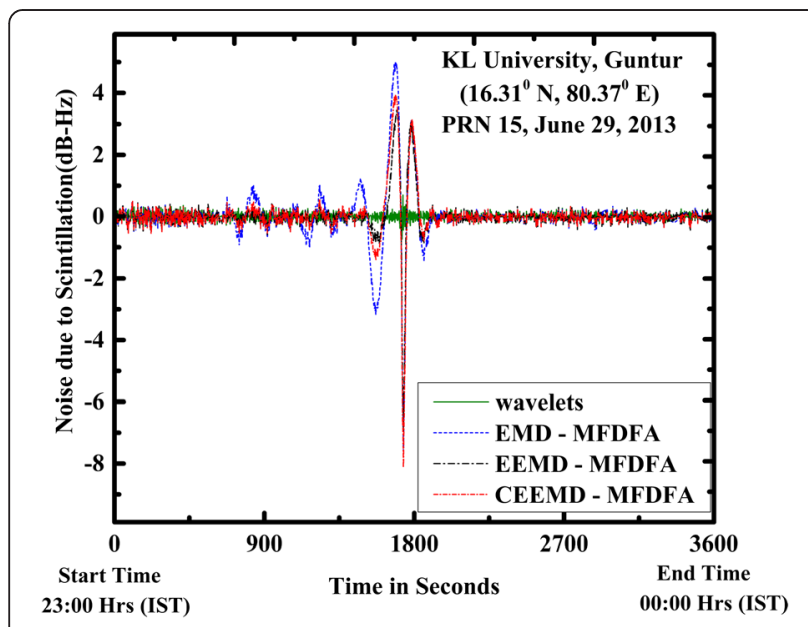

Fig. 3 The results of the CEEMD-MF-DFA method in reducing the noise from the GPS signal the CEEMD-MF-DFA method performed well in the mitigation of ionospheric scintillations.

The performance of the CEEMD-MF-DFA was compared and validated with the results of the wavelet, EMDMF-DFA and EEMD-MF-DFA methods, as shown in Fig. 4. Daubechies wavelet of order 8 was used along with a soft threshold. It is evident that the mitigation of scintillation noise is better with the CEEMD-MF-DFA technique than the wavelet, EMD-MF-DFA and EEMD-MF-DFA methods. The $C / N_{0}$ value was improved to $39.20 \mathrm{~dB}-\mathrm{Hz}$ using the proposed algorithm corresponding to $31 \mathrm{~dB}-\mathrm{Hz}$ of the original signal at $23.28 \mathrm{~h}$ (IST) on 29 June 2013 for PRN 15 when fluctuation order $q=5$ was used. The corresponding $C / N_{0}$ values for wavelets, EMD and EEMD were 31.44, 38.64 and $38.03 \mathrm{~dB}-\mathrm{Hz}$, respectively. More than $1.1 \mathrm{~dB}-\mathrm{Hz}$ of scintillation is extracted by the proposed method as compared to the EEMD-MF-DFA technique.

\section{Conclusions}

A new algorithm based on CEEMD-MF-DFA was implemented for mitigating the noise components due to ionospheric scintillations in GNSS signals. The performance of the proposed method was compared with the results of the wavelet, EMD-MF-DFA and EEMD-MF-DFA methods. The IMFs obtained using EMD, EEMD and CEEMD for $C / N_{0}$ signal were applied to the MF-DFA method to calculate the Hurst exponent, which is essential for estimating the threshold for detecting the noise due to scintillation. $8.20 \mathrm{~dB}-\mathrm{Hz}$ of noise was detected and reduced using CEEMD-MF-DFA. It is evident from the results that more than $1.1 \mathrm{~dB}-\mathrm{Hz}$ noise was filtered out by the proposed method as compared to other techniques. The results will be useful for understanding the morphology of non-linear ionospheric irregularities.

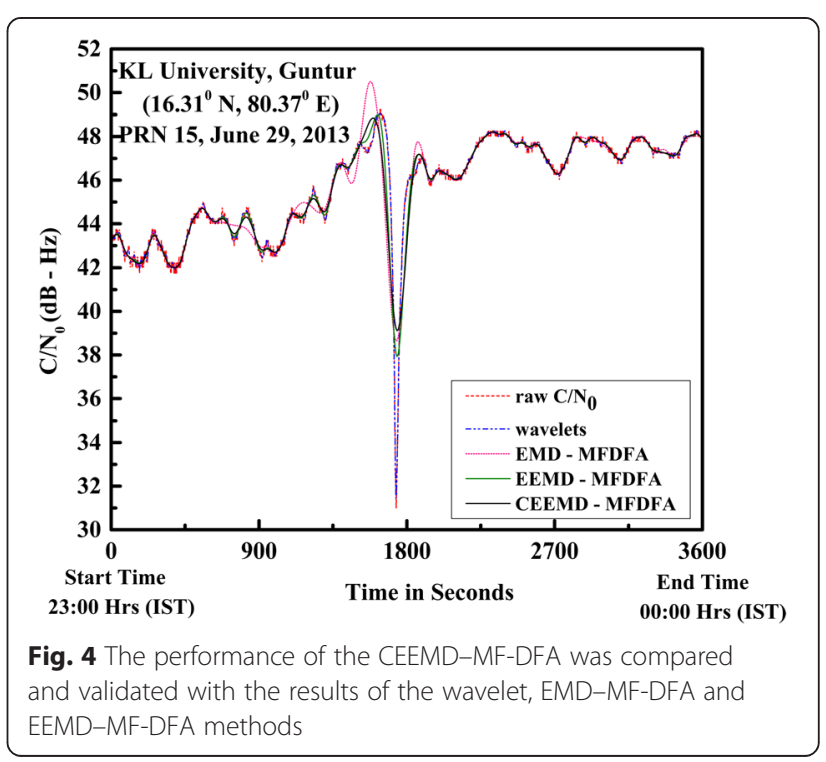




\section{Competing interests}

The authors declare that they have no competing interests.

\section{Authors' contributions}

SM collected the data sets for the last 2 years and drafted the manuscript SRC analysed the data and identified the scintillation events for which the multifractal analysis is applied. PRK verified the results and discussed them with all the authors. All authors read and approved the final manuscript.

\section{Authors' information}

S. Miriyala is presently working as an associate professor in the Department of ECE, KL University, Guntur, Andhra Pradesh, India. P. R. Koppireddi is a professor in the Department of ECE, JNTU, Kakinada, and presently, he is working as the principal of the University College of Engineering, JNTU Kakinada, Kakinada, Andhra Pradesh, India. S. R. Chanamallu is a professor in the Department of ECE and presently working as the Head of the Department, Department of ECE, JNTU Kakinada, Vizianagaram, Andhra Pradesh, India.

\section{Acknowledgements}

The above work has been carried out under the project titled "Development of Ionospheric Forecasting models for Satellite based Navigation Systems over low latitude stations" sponsored by the Department of Science and Technology, New Delhi, India, vide sanction letter No: SR/FTP/ETA-0029/2012, dated: 08.05.12. The authors are very much thankful to Dr. D. Venkata Ratnam, Professor, KL University, for his outstanding guidance in the work and cooperation.

\section{Author details}

${ }^{1}$ Department of ECE, KL University, Guntur, India. ²Department of ECE, JNTUK, Kakinada, India. ${ }^{3}$ Department of ECE, JNTUK, Vizianagaram, India.

Received: 22 April 2015 Accepted: 9 June 2015

Published online: 24 June 2015

\section{References}

Aquino M, Sreeja V (2013) Correlation of scintillation occurrence with interplanetary magnetic field reversals and impact on Global Navigation Satellite System receiver tracking performance. Space Weather 11(5):219-224

Cherniak I, Zakharenkova I, Krankowski A (2014) Approaches for modeling ionosphere irregularities based on the TEC rate index. Earth, Planets Space 66(1):1-5

De Paula ER, Rodrigues FS, Iyer KN, Kantor IJ, Abdu MA, Kintner PM, Ledvina BM, Kil H (2003) Equatorial anomaly effects on GPS scintillations in Brazil. Adv Space Res 31(3):749-754

Huang NE, Shen Z, Long SR, Wu MC, Shih HH, Zheng Q, Yen NC, Tung CC, Liu H (1998) The empirical mode decomposition and the Hilbert spectrum for nonlinear and non-stationary time series analysis. Proc R Soc Lond Series A 454:903-995

Hurst HE (1951) Long-term storage of reservoirs. T Am Soc Civ Eng 116:770-808

Ihlen EA (2012) Introduction to multifractal detrended fluctuation analysis in Matlab. Front Physiol 3:141

lyer KN, Souza JR, Pathan BM, Abdu MA, Jivani MN, Joshi HP (2006) A model of equatorial and low latitude VHF scintillation in India. Indian J Radio Space Physics 35(2):98

Kantelhardt JW, Zschiegner SA, Bunde A, Havlin S, Koscielny-Bunde E, Stanley HE (2002) Multifractal detrended fluctuation analysis of nonstationary time series. Physica A 316:87

Kintner PM, Ledvina BM, De Paula ER (2007) GPS and ionospheric scintillations. Space Weather 5:S09003. doi:10.1029/2006SW000260

Mallat S (1999) A wavelet tour of signal processing. Academic Press

Mert A, Akan A (2014) Detrended fluctuation thresholding for empirical mode decomposition based denoising. Digit Signal Process 32:48-56

Mushini SC, Jayachandran PT, Langley RB, MacDougall JW, Pokhotelov D (2012) Improved amplitude and phase-scintillation indices derived from wavelet detrended high-latitude GPS data. GPS Solutions 16(3):363-373

Pullen S, Park YS, Enge P (2009) Impact and mitigation of ionospheric anomalies on ground-based augmentation of GNSS. Radio Sci 44 (1)

Suman G, Jovancevic A, Brown A, Kirchner M, Zigic S, Beach T, Groves KM (2004) lonospheric scintillation monitoring and mitigation using a software GPS receiver. Radio Science 39 (1)
Tanna HJ, Pathak KN (2014) Multifractality due to long-range correlation in the L-band ionospheric scintillation S 4 index time series. Astrophys Space Sci 350(1):47-56

Venkata Ratnam D, Sivavaraprasad G, Lee J (2015) Automatic ionospheric scintillation detector for global navigation satellite system receivers. IET Radar, Sonar Navigation. doi:10.1049/iet-rsn.2014.0232

Wang T, Zhang M, Yu Q, Zhang H (2012) Comparing the applications of EMD and EEMD on time-frequency analysis of seismic signal. J Appl Geophys 83:29-34

Wernik AW, Alfonsi L, Materassi M (2004) lonospheric irregularities, scintillation and its effect on systems. Acta Geophysica Polonica 52(2):237-249

Wu Z, Huang NE (2009) Ensemble empirical mode decomposition: a noiseassisted data analysis method. Advances in adaptive data analysis 1(01):1-41

Yeh KC, Liu CH (1982) Radio wave scintillations in the ionosphere. Proc IEEE 70(4):324-360

Yeh JR, Shieh JS, Huang NE (2010) Complementary ensemble empirical mode decomposition: a novel noise enhanced data analysis method. Adv Adapt Data Anal 2(02):135-156

\section{Submit your manuscript to a SpringerOpen ${ }^{\circ}$ journal and benefit from:}

- Convenient online submission

Rigorous peer review

- Immediate publication on acceptance

- Open access: articles freely available online

- High visibility within the field

- Retaining the copyright to your article

Submit your next manuscript at $>$ springeropen.com 